\title{
Efficient Nonlinear Light Emission of Single Gold Optical Antennas Driven by Few-Cycle Near-Infrared Pulses
}

\author{
T. Hanke, G. Krauss, D. Träutlein, B. Wild, R. Bratschitsch, and A. Leitenstorfer \\ Department of Physics and Center for Applied Photonics, University of Konstanz, D-78457 Konstanz, Germany \\ (Received 31 July 2009; published 17 December 2009)
}

\begin{abstract}
Individual nanometer-sized plasmonic antennas are excited resonantly with few-cycle laser pulses in the near infrared. Intense third-harmonic emission of visible light prevails for fundamental photon energies below $1.1 \mathrm{eV}$. Interband luminescence and second harmonic generation occur solely at higher driving frequencies. We attribute these findings to multiphoton resonances with the $d$-band transitions of gold. The strong third-order signal allows direct measurement of a subcycle plasmon dephasing time of $2 \mathrm{fs}$, highlighting the efficient radiation coupling and broadband response of the devices.
\end{abstract}

DOI: 10.1103/PhysRevLett.103.257404

Metal nanoantennas $[1,2]$ have recently been demonstrated to work as optical analogues to their ubiquitous counterparts at radio and microwave frequencies. They have been used to couple visible radiation to single nanoscopic light emitters such as semiconductor quantum dots [3] or molecules [4]. Although the linear response of optical antennae has been investigated in detail [5-8], little is yet known about their nonlinear properties. Emission attributed to coherent supercontinuum and high harmonics generation [1,9], as well as incoherent two-photon photoluminescence $[2,10]$ have been observed. Up to now, experiments were performed by exciting nanoantennae with ultrashort laser pulses originating from Ti:sapphire lasers operating at photon energies around $1.5 \mathrm{eV}$. Typically, large arrays of antennas have been employed to overcome small conversion efficiencies. In this work, we exploit ultrabroadband Er:fiber technology delivering tunable laser pulses in the few-cycle regime [11] to investigate single gold nanoantennae under excitation in the near infrared. We observe a variety of strong nonlinear signals in the visible spectral range. The efficiencies depend critically on the photon energy of the excitation.

Well-defined arrays of linear, bowtie, and elliptically shaped structures are fabricated via electron beam lithography on fused silica substrates. The thicknesses of the gold film, chromium adhesion layer, and dielectric substrate amount to $40 \mathrm{~nm}, 2 \mathrm{~nm}$, and $170 \mu \mathrm{m}$, respectively. Antenna size and gap dimensions are systematically varied. The data featured here represent fundamental phenomena observed for all antenna geometries under investigation.

Our femtosecond Er:fiber system operates at a repetition rate of $40 \mathrm{MHz}$. It delivers pulses of a duration of $7.8 \mathrm{fs}$ with a spectral bandwidth extending from 0.85 to $1.4 \mathrm{eV}$ [11]. To single out individual nanoantennae, we focus to a spot diameter of $2 \mu \mathrm{m}$ via all-reflective optics, minimizing dispersion and achromatism. The linear polarization of the excitation is set parallel to the main antenna axis. The emission is collected in transmission geometry with an
PACS numbers: 78.67.Uh, 42.65.Ky, 73.20.Mf, 78.47.Fg

objective lens of a numerical aperture of $\mathrm{NA}=0.55$. Antenna samples may be raster scanned relative to the confocal region with a piezoelectric translation stage. Figure 1 shows the spectrally integrated visible emission as a function of lateral position in an array of 48 linear nanoantennae of varying arm length $l$ and feed gap $g$. We excite with the ultrabroadband spectrum centered at a wavelength of $1.2 \mu \mathrm{m}$ and an average intensity set to $0.6 \mathrm{MW} / \mathrm{cm}^{2}$. Note that the blue light emanating from

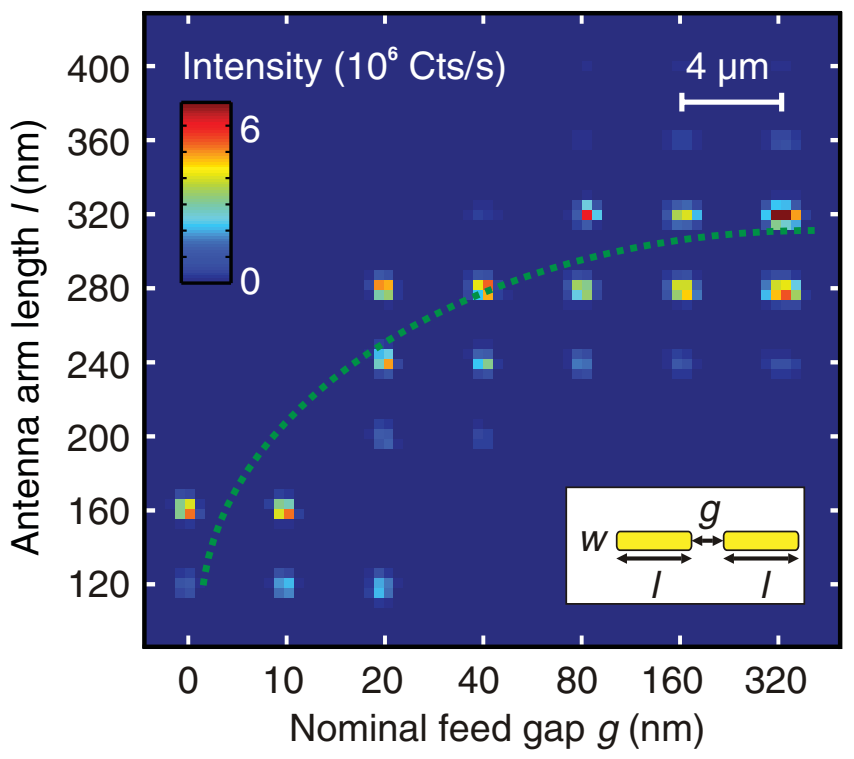

FIG. 1 (color). Spectrally integrated nonlinear emission from 48 individual linear gold antennae of different arm length $l$ and feed gap $g$. The arm width $w$ is kept constant at $60 \mathrm{~nm}$. Lateral and vertical distances between antennas are set to $4 \mu \mathrm{m}$ and $3 \mu \mathrm{m}$, respectively. The nanostructures are raster scanned through the focus of a 7.8-fs laser pulse with a spectrum extending from 0.85 to $1.4 \mathrm{eV}$. The visible intensity is depicted in a color scale as a function of position. The dashed line is a guide to the eye illustrating the regions with antennae in resonance with the driving field. Inset: schematic drawing of the antenna geometry studied in this data set. 
individual nanoantennae is easily visible with the bare human eye [12]. The average power generated by the brightest devices is in the order of $0.7 \mathrm{nW}$. For structures with feed gaps wider than $g=40 \mathrm{~nm}$, high emission intensities are observed when the excitation bandwidth includes wavelengths corresponding to a quarter of a single antenna arm, i.e., for $l \approx 300 \mathrm{~nm}[6,7]$. As soon as the two arms begin to couple electrodynamically for feed gaps $g$ around $40 \mathrm{~nm}$, the redshift of the dipole resonance [13] leads to resonant conditions at shorter overall antenna lengths (see dashed line in Fig. 1). This shift finally evolves into the $\lambda / 4$ resonance of a single rod at the onset of close contact of the two antenna arms (nominal feed gaps $g$ of $0 \mathrm{~nm}$ and $10 \mathrm{~nm}$ ). The observation of this systematic behavior in the antenna array demonstrates the quality of the fabrication process and the reproducibility of our structures.

To spectrally resolve the radiation from each nanoantenna, we use a monochromator and a Peltier-cooled CCD camera. Figure 2 depicts a typical nonlinear emission spectrum from a single bowtie resonant device excited at a pump intensity of $0.5 \mathrm{MW} / \mathrm{cm}^{2}$. We observe strong third-harmonic generation (THG) together with a weak second harmonic signal (SHG). The spectral positions of THG and SHG point to the same fundamental frequency determined by the plasmon resonance of the metal nanostructure in the near infrared. We find a cubic and quadratic dependence of the emission intensity on excitation power for the THG and SHG, respectively. The high intensity of

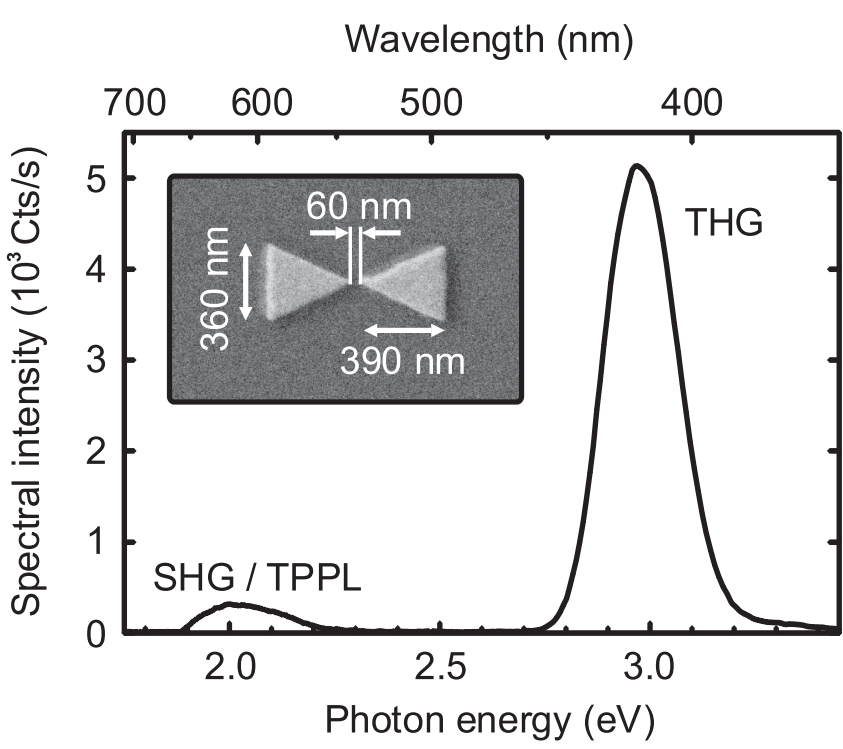

FIG. 2. Typical nonlinear emission spectrum from a single gold optical antenna excited with the total bandwidth of the 7.8-fs pulse. SHG: second harmonic generation, TPPL: twophoton photoluminescence, THG: third-harmonic generation. Inset: scanning electron microscope image of the antenna. A bowtie-shaped geometry is featured in this figure which is composed of two opposing nanotriangles (triangle height: $390 \mathrm{~nm}$, triangle base: $360 \mathrm{~nm}$, gap: $60 \mathrm{~nm}$ ). the third harmonic may be attributed to the strong $\chi^{(3)}$ nonlinearity of $\mathrm{Au}$ [14]. The smaller efficiency for SHG is consistent with the centrosymmetric character of bulk gold and fused silica [15]. In the forward direction, frequency doubling might occur solely due to hyper-Rayleigh scattering at surface imperfections [16]. Most likely, the SHG we observe is due to excitation of nonlocal electric dipole and local electric quadrupole modes [17] or electromagnetic effects in the confined plasma system [18]. In our geometry, these components get collected due to the high numerical aperture of our detection optics.

To elaborate the microscopic origin of the nonlinear emission components we tune the photon energy of the pump while keeping the antennas resonant by proper control of geometry: A strong third-harmonic emission is observed for excitation with a 20 -fs laser pulse centered at $1 \mathrm{eV}$ [Fig. 3(a)]. When the driving pulse contains photon energies beyond $1.1 \mathrm{eV}$, we obtain a signal at the second harmonic frequency [see Fig. 3(b)]. At the same time, the SHG peak develops a low-energy shoulder. We attribute this feature to two-photon photoluminescence (TPPL). This component is known from experiments with Ti:sapphire lasers $[2,19]$. It has been used previously to map the electric field around single metal nanoantennas [10]. A tentative explanation for the drastic change of the dominating nonlinear process when tuning the excitation above $1.1 \mathrm{eV}$ is motivated by the synchronous appearance of SHG and TPPL: As long as the photon energy is too low

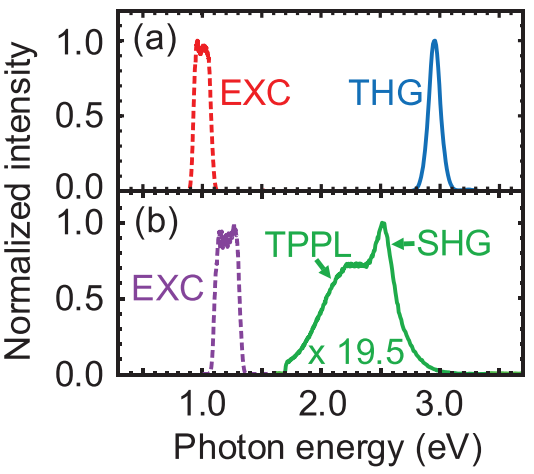

(c)

(d)
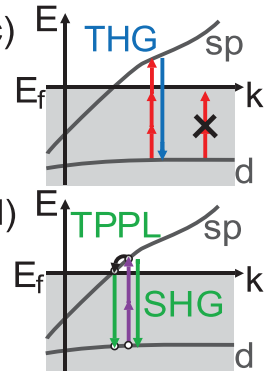

FIG. 3 (color). Nonlinear emission from resonant gold nanoantennas depending on excitation photon energy. (a) Thirdharmonic emission (THG, blue line) of a linear device with arm length $l=320 \mathrm{~nm}$, width $w=60 \mathrm{~nm}$ and feed gap $g=$ $40 \mathrm{~nm}$, excited with a pump spectrum (EXC) confined below $1.1 \mathrm{eV}$ (dashed red line). (b) The two-photon photoluminescence (TPPL) and second harmonic generation (SHG) from a specimen with $l=240 \mathrm{~nm}, w=60 \mathrm{~nm}$ and $g=40 \mathrm{~nm}$, excited with a pump photon energy above $1.1 \mathrm{eV}$ (magenta dashed line) is depicted as a green line. The peak spectral intensity of the TPPL/ SHG signal is weaker by a factor of 19.5 as compared to the THG emission in (a). (c) and (d) Sketches of the band structure of gold illustrating the relevant pump and emission processes for the conditions in (a) and (b), respectively. Arrow lengths indicate photon energies in excitation (up) and emission (down). Colors correspond to the processes indicated in (a) and (b). 
to induce two-photon transitions of electrons out of the $d$-bands across the Fermi energy and into the empty states in the $s p$-type conduction band, the antenna response is dominated by the strong third-order nonlinearity and a clean THG signal is emitted. This situation is sketched in the band structure scheme of Fig. 3(c). As soon as the twophoton resonance of the $d$-band transition is reached, TPPL sets in and at the same time, second-order nonlinear effects may be resonantly enhanced [see Fig. 3(d)]. Interestingly, the THG emission from single antennae becomes hardly discernible for pump photon energies beyond $1.1 \mathrm{eV}$, although it falls well into our detection window [see Fig. 3(b)]. The explanation for this behavior may be found in a combination of two effects: (i) THG may be enhanced at lower driving frequencies due to a three-photon resonance with the $d$-band transitions of bulk Au [see Fig. 3(c)]. (ii) The two-photon resonance occurring at shorter pump wavelengths [Fig. 3(d)] may render the third-order nonlinear response predominantly imaginary, effectively quenching THG which is linked to the real part of $\chi^{(3)}$.

The efficient THG emission we find for pump photon energies below $1.1 \mathrm{eV}$ is now exploited to directly measure the ultrafast plasmon dephasing time of a single nanoantenna. To this end, we excite collinearly with two identical transients derived from a Mach-Zehnder interferometer and record the emitted THG signal as a function of time delay between the pulses. Frequency-resolved optical gating (FROG) measurements [20-22] are performed with the spectrometer and the CCD camera. To achieve the highest time resolution available combined with an unchirped driving field, we restrict the spectrum of the excitation pulse to the region where it features a flat phase relationship. This strategy results in a temporal duration close to the bandwidth limit and a clean pulse shape. We characterize the transient by recording a FROG trace from the nonresonant signal generated with the bare fused silica surface next to the antenna structure. Figure 4(a) shows the emitted THG intensity color coded as a function of delay time and frequency position. The interference fringes due to the collinear geometry were removed via Fourier filtering. Careful FROG analysis [23] yields a pulse duration of $9.8 \mathrm{fs}$, a spectral bandwidth of $51 \mathrm{THz}$ and a carrier frequency of $248 \mathrm{THz}$, equivalent to a central wavelength of $1210 \mathrm{~nm}$. The accuracy of our characterization is demonstrated by the close coincidence of the measured third-harmonic autocorrelation (dotted line in Fig. 4(c)] with the trace calculated from the amplitude and phase spectra obtained via FROG (red line in Fig. 4(c)]. With exactly the same pump pulse we now resonantly excite an elliptical Au nanoantenna. The Fourier-filtered FROG data are depicted in Fig. 4(b). Note that the THG intensity is 2 orders of magnitude higher than from the bare substrate. The spectral full width at half maximum (FWHM) of the signal decreases from

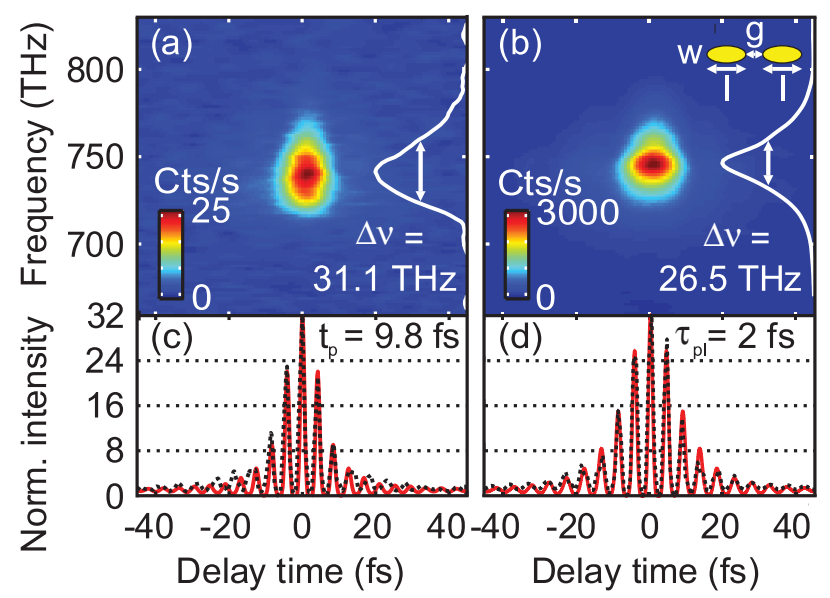

FIG. 4 (color). Dephasing of a single resonantly driven gold nanoantenna: Fourier-filtered third-harmonic emission intensity as a function of delay time and frequency for (a) the bare fused silica substrate and (b) an elliptical gold nanoantenna (arm length $l=250 \mathrm{~nm}$, width $w=100 \mathrm{~nm}$, and feed gap $g=$ $40 \mathrm{~nm}$ ). Integrated emission spectra are depicted as white lines. Interferometric third-order autocorrelation traces from (c) the substrate and (d) the resonant antenna. Careful analysis and comparison of the experimental data (dotted lines) with simulations (red lines) yield a reference pulse duration of $t_{p}=9.8 \mathrm{fs}$ and a plasmon dephasing time of $\tau_{\mathrm{pl}}=2.0 \mathrm{fs}$.

31.1 THz in the nonresonant measurement of Fig. 4(a) to 26.5 THz for the antenna emission in Fig. 4(b). Correspondingly, the temporal width of the spectrally integrated third-order autocorrelation [dotted line in Fig. 4(d)] increases significantly as compared to the instantaneous response of the substrate [Fig. 4(c)]. To quantify the plasmon dephasing of the nanoantenna system, we fit the interferometric autocorrelation by assuming a fundamental plasmon oscillator with an exponential damping term and a perturbative third-order nonlinearity [24]. The driving electric field is retrieved from the FROG trace in Fig. 4(a). If this method is applied integrating over an array of metal nanostructures, a lower limit for the individual dephasing times is obtained due to the potential influence of inhomogenous broadening [24]. In contrast, our study of a single specimen gives direct access to the precise value of the damping constant. The THG autocorrelation simulated assuming a plasmon dephasing time of $2 \mathrm{fs}$ is depicted as a red line in Fig. 4(d). It is in excellent agreement with the experimental result [dotted line in Fig. 4(d)]. By comparison with simulated autocorrelation traces for $1.5 \mathrm{fs}$ and 2.5 fs dephasing times, we estimate the experimental error of this measurement to be as small as \pm 500 attoseconds.

The ultrashort dephasing of our antennae is clearly dominated by radiation damping [25-27]. Note that a damping time of $2 \mathrm{fs}$ corresponds to less than an oscillation cycle of the fundamental plasmon resonance of the antenna of $250 \mathrm{THz}$. In the context of metal nanoparticles, it has been argued that long plasmon dephasing times are a 
prerequisite for the occurrence of strong field enhancement and efficient nonlinear optics [26,27]. Nevertheless, we obtain an increase of the resonant nonlinear emission of more than an order of magnitude as compared to offresonant driving (see Fig. 1). Obviously, the relevance of long plasmon dephasing times for the efficiency of nonlinear processes is limited when working with optical antennae. In fact, these are devices where radiation energy is collected from a large cross-sectional area and funneled into a small volume near the antenna gap where a strong field enhancement arises. Since electromagnetic coupling works in both directions, optimized antenna geometries will always tend to come with relatively fast radiation damping. Therefore, these systems are naturally compatible with few-femtosecond optical excitation, providing the efficient harmonics generation we find under resonant conditions.

In conclusion, we have studied the nonlinear optical properties of single gold nanoantennae driven with fewcycle laser pulses in the near infrared. Intense thirdharmonic emission is obtained when exciting with fundamental spectra below $1.1 \mathrm{eV}$. At higher photon energies, additional components corresponding to frequency doubling and two-photon induced luminescence are observed together with a fading of THG. We relate these findings to the band structure of bulk Au, especially to a two-photon resonance with the $d$-band transitions. A plasmon dephasing as fast as $2 \mathrm{fs}$ is directly measured in the time domain. We expect the bright third-harmonic emission to originate from a volume of a few $(10 \mathrm{~nm})^{3}$ close to the feed gap of our antennae. Therefore, they enable selective excitation and sensitive probing of optical properties in nanoscale spatial regions with femtosecond time resolution and ultrabroadband spectral coverage. Consequently, such structures will be attractive tools for new classes of experiments like femtosecond quantum optics with solidstate single-electron systems [28]. Pumped with a few $\mathrm{mW}$ from compact fiber lasers, the intensive and coherent nanometer-sized light sources might also find technical applications, e.g., in readout devices for ultrahigh density optical data storage.

[1] P. Mühlschlegel, H.-J. Eisler, O. J. F. Martin, B. Hecht, and D. W. Pohl, Science 308, 1607 (2005).

[2] P. J. Schuck, D.P. Fromm, A. Sundaramurthy, G. S. Kino, and W.E. Moerner, Phys. Rev. Lett. 94, 017402 (2005).

[3] J. N. Farahani, D. W. Pohl, H.-J. Eisler, and B. Hecht, Phys. Rev. Lett. 95, 017402 (2005).

[4] T. H. Taminiau, R. J. Moerland, F. B. Segerink, L. Kuipers, and N. F. van Hulst, Nano Lett. 7, 28 (2007).
[5] L. Rogobete, F. Kaminski, M. Agio, and V. Sandoghdar, Opt. Lett. 32, 1623 (2007).

[6] G. W. Bryant, F. J. Garcia de Abajo, and J. Aizpurua, Nano Lett. 8, 631 (2008).

[7] L. Novotny, Phys. Rev. Lett. 98, 266802 (2007).

[8] J. Merlein, M. Kahl, A. Zuschlag, A. Sell, A. Halm, J. Boneberg, P. Leiderer, A. Leitenstorfer, and R. Bratschitsch, Nat. Photon. 2, 230 (2008).

[9] S. Kim, J. Jin, Y.-J. Kim, I.-Y. Park, Y. Kim, and S.-W. Kim, Nature (London) 453, 757 (2008).

[10] P. Ghenuche, S. Cherukulappurath, T. H. Taminiau, N. F. van Hulst, and R. Quidant, Phys. Rev. Lett. 101, 116805 (2008).

[11] A. Sell, G. Krauss, R. Scheu, R. Huber, and A. Leitenstorfer, Opt. Express 17, 1070 (2009).

[12] See EPAPS Document No. E-PRLTAO-104-009001 for a real-time video of THG emission of antennas scanned through the excitation focus.

[13] J. Aizpurua, G. W. Bryant, L. J. Richter, F. J. Garcia de Abajo, B. K. Kelley, and T. Mallouk, Phys. Rev. B 71, 235420 (2005).

[14] M. Lippitz, M. A. van Dijk, and M. Orrit, Nano Lett. 5, 799 (2005).

[15] B. K. Canfield, H. Husu, J. Laukkanen, B. Bai, M. Kuittinen, J. Turunen, and M. Kauranen, Nano Lett. 7, 1251 (2007).

[16] M. I. Stockman, D. J. Bergman, C. Anceau, S. Brasselet, and J. Zyss, Phys. Rev. Lett. 92, 057402 (2004).

[17] J. I. Dadap, J. Shan, K. B. Eisenthal, and T. F. Heinz, Phys. Rev. Lett. 83, 4045 (1999).

[18] Y. Zeng, W. Hoyer, J. Liu, S. W. Koch, and J. V. Moloney, Phys. Rev. B 79, 235109 (2009).

[19] A. Bouhelier, R. Bachelot, G. Lerondel, S. Kostcheev, P. Royer, and G. P. Wiederrecht, Phys. Rev. Lett. 95, 267405 (2005).

[20] T. Tsang, M. A. Krumbügel, K. W. DeLong, D. N. Fittinghoff, and R. Trebino, Opt. Lett. 21, 1381 (1996).

[21] G. Stibenz and G. Steinmeyer, Opt. Express 13, 2617 (2005).

[22] A. Anderson, G. Steinmeyer, and M. B. Raschke, Conference on Lasers and Electro-Optics/International Quantum Electronics Conference, OSA Technical Digest (CD) (Optical Society of America, Washington, DC, 2009), paper CThK2

[23] The FROG error on a grid size of 128 amounts to 0.015 .

[24] B. Lamprecht, J.R. Krenn, A. Leitner, and F. R. Aussenegg, Phys. Rev. Lett. 83, 4421 (1999).

[25] A. Wokaun, J. P. Gordon, and P. F. Liao, Phys. Rev. Lett. 48, 957 (1982).

[26] C. Sönnichsen, T. Franzl, T. Wilk, G. von Plessen, J. Feldmann, O. Wilson, and P. Mulvaney, Phys. Rev. Lett. 88, 077402 (2002).

[27] T. Klar, M. Perner, S. Grosse, G. von Plessen, W. Spirkl, and J. Feldmann, Phys. Rev. Lett. 80, 4249 (1998).

[28] F. Sotier, T. Thomay, T. Hanke, J. Korger, S. Mahapatra, A. Frey, K. Brunner, R. Bratschitsch, and A. Leitenstorfer, Nature Phys. 5, 352 (2009). 